\title{
UTILIZACIÓN DE ESCALAS GRADUADAS PARA AUTOEVALUAR Y COEVALUAR: UNA EXPERIENCIA DE AULA EN LA EDUCACIÓN VIRTUAL SUPERIOR
}

Use of graduated scales to self-assessment and peer-assessment: a classroom experience in higher virtual education.

Uso de escalas graduadas para autoavaliar e coavaliar: uma experiência em sala de aula no ensino virtual superior.

\section{Ivana Marsicano (1)}

(1) Universidad Tecnológica, Uruguay. Teléfono: +59899220986 . Correo electrónico: ivana.marsicano@utec.edu.uy

\section{Resumen}

En el marco del desarrollo de un curso virtual, autoadministrado, a través de la plataforma Open edX, se llevó a cabo una propuesta de evaluación formativa denominada "Trabajo Final Enriquecido por Pares" que se realizó a través de una autoevaluación y una coevaluación para las que se facilitó una escala graduada que fue compartida desde el inicio del curso junto con los criterios de aprobación. En este trabajo se describen todas las etapas de la propuesta, así como las valoraciones de los participantes al respecto de su utilidad tanto para el enriquecimiento del trabajo, como para la adquisición del aprendizaje. Los resultados muestran que el conocimiento de los criterios de valoración del trabajo final a través de la escala graduada y de los del curso, permitieron reducir la ansiedad $\mathrm{y}$ fortalecer los procesos de autoevaluación $\mathrm{y}$ coevaluación lo que enriqueció tanto la producción del trabajo, como la adquisición del aprendizaje.

Palabras clave: Educación Virtual, Evaluación Formativa, Coevaluación, Autoevaluación, Escalas Graduadas

\begin{abstract}
Within the framework of the development of a self-administered virtual course, through the Open edX platform, a formative assessment proposal named "Trabajo Final Enriquecido por Pares" (meaning "Final Work enriched by Peers"), was carried out
\end{abstract}


through a self-assessment and a co-assessment for which a graded scale was provided that was shared from the beginning of the course along with the approval criteria. In this work, all the stages of the proposal are described, as well as the participant's evaluations of its usefulness both for the enrichment of the work and for the acquisition of learning. The results show that the knowledge of both the assessment criteria of the course and the Final Work through the graduated scale gave a transparency in the way of assessment that allowed the students to reduce the anxiety and to strengthen the process of peer-assessment and self-assessment. As a consequence, the production of work was enriched, and so was the acquisition of learning.

Keywords: Virtual Education, Formative Assessment, Peer-assessment, Selfassessment, Graduated Scales

\section{Resumo}

No âmbito do desenvolvimento de um curso virtual, autoadministrado, através da plataforma Open edX, foi realizada uma proposta de avaliação formativa chamada "Trabalho Final Enriquecido por Pares", através da entrega de uma escala graduada que facilitou o uso da autoavaliação e da coavaliação e que foi partilhada desde o início do curso, juntamente com os critérios de avaliação. Neste trabalho, são descritas todas as etapas da proposta, bem como as avaliações dos participantes quanto à sua utilidade tanto para o enriquecimento do trabalho como para a aquisição da aprendizagem. Os resultados mostram que o conhecimento dos critérios de avaliação do trabalho final, por meio da escala graduada e do curso, permitiu reduzir a ansiedade e fortalecer os processos de autoavaliação e coavaliação, o que enriqueceu tanto a produção do trabalho como a aquisição da aprendizagem.

Palavras-chave: Educação Virtual, Avaliação Formativa, Coavaliação, Autoavaliação, Escalas Graduadas

\section{Introducción}

El Programa de Evaluación y Estadística de la Universidad Tecnológica de Uruguay es uno de los programas que integran el Departamento de Programas Especiales, el cual lleva adelante propuestas transversales a todas las carreras de la universidad para el desarrollo de habilidades y competencias del siglo XXI, ofreciendo actividades con 
créditos académicos, de libre elección por parte de los estudiantes y configuración como propuesta docente, calificadas a través de una devolución cualitativa con una resolución de suficiencia o insuficiencia.

Bajo este marco, la implementación de actividades que fomenten el aprendizaje autónomo y autorregulado está íntimamente ligado al uso de la evaluación formativa como potenciadora de los procesos de enseñanza y aprendizaje. La autoevaluación y la coevaluación son estrategias de evaluación formativa que propician el involucramiento de los estudiantes y potencian la autonomía y autorregulación (Gómez-Ruiz y Quesada, 2017; Marsicano, 2018). Por su parte, el uso de escalas graduadas (EG), que permiten evaluar la globalidad de un trabajo identificando fortalezas y debilidades, ha sido valorado positivamente por los estudiantes tanto para guiar la elaboración de un trabajo como para que su logro sea de mayor calidad (López-Pastor, Pérez-Pueyo, Barba y Lorente-Catalán, 2016).

A continuación, se comparte una experiencia educativa llamada "Trabajo Final Enriquecido por Pares" (TFEpP) junto a las valoraciones de los estudiantes.

\section{Contextualización}

El curso en donde se implementó esta experiencia, en modalidad virtual a través de la plataforma Open edX, tuvo una carga horaria de 45 horas. Para su aprobación se requirió: (a) la realización correcta de por lo menos el 50\% de las actividades de respuesta inmediata propuestas en plataforma; (b) la entrega y la obtención de suficiencia en el TFEpP que se efectivizó a través de una doble entrega, una primera junto a una autoevaluación para ser evaluado por un par con una EG, y otra luego de ser revisado y ajustado considerando la devolución del par para ser evaluado por el docente con la misma EG; (c) la participación en la coevaluación.

La práctica TFEpP se llevó a cabo con una muestra de 32 estudiantes. Se diseñó "ad hoc" una EG como instrumento de evaluación del trabajo final que permitiera realizar evaluación formativa durante la elaboración, autoevaluación, coevaluación y corrección de los trabajos. Los trabajos fueron de elaboración individual bajo una consigna previamente acordada.

La intención de facilitar una EG fue doble, por un lado, para dar a conocer desde el inicio los aspectos y criterios que se utilizarían para evaluar el trabajo final y, por otro lado, para que los participantes pudiesen autoevaluarse y coevaluarse en los mismos 
aspectos y con los mismos criterios. Para ello, en la escala se establecieron 15 horquillas que describían cada uno de los aspectos a evaluar, en las siguientes opciones: Necesita Mejorar Mucho (Insuficiente); Necesita Mejoras (Insuficiente); Correcto (Suficiente); Es muy bueno (Suficiente); Es excelente (Suficiente).

Se le agregó una columna de "Devolución" a la EG ya que se incluyó una devolución a cada marca asignada, de manera de aportar insumos en la mejora del trabajo. La escala fue calibrada luego de haber sido enviada a dos profesores expertos y testada con 5 estudiantes.

\section{Diseño y desarrollo}

La aplicación de la experiencia se realizó en las siguientes etapas: (a) Socialización de criterios de aprobación y EG; (b) Elaboración y Entrega de la primer versión del TFEpP y realización de autoevaluación haciendo uso de la EG; (c) Coevaluación entre pares haciendo uso de la EG e incluyendo una devolución en cada marca; (d) Reformulación del TFEpP en función de la coevaluación realizada y recibida; (e) Entrega final del TFEpP; (f) Devolución docente; (g) Formulario de Evaluación de la Experiencia.

Para recoger las valoraciones se aplicó un cuestionario anónimo, elaborado "ad hoc", con 8 preguntas cerradas en una escala de Likert de 5 puntos y 2 preguntas abiertas.

El formulario fue enviado a dos profesores expertos y testado en un piloto con 5 estudiantes.

\section{Evaluación y conclusiones}

En la Tabla 1 se muestran los aspectos relevados junto a las valoraciones medias y modas que le asignaron los participantes a cada uno de éstos.

Tabla 1.

Media y Moda de las valoraciones de los participantes en escala de Likert 5 puntos: 1 Nada, 5 Mucho.

\begin{tabular}{lcc}
\hline Aspectos & Media & Moda \\
\hline $\begin{array}{l}\text { Haber conocido los criterios de valoración del curso y del TFEpP para el } \\
\text { enriquecimiento del trabajo. }\end{array}$ & 4,5 & 5 \\
Haber conocido los criterios de valoración del curso y del TFEpP para la & 4,1 & 4 \\
adquisición del aprendizaje. & 4,2 & 4 \\
Haber realizado una coevaluación para el enriquecimiento del trabajo. & 4,3 & 4 \\
Haber realizado una coevaluación para la adquisición del aprendizaje. & 4,7 & 5 \\
Haber recibido una coevaluación para el enriquecimiento del trabajo. & 4,6 & 5 \\
Haber recibido una coevaluación para la adquisición del aprendizaje. & 4,6 & 5 \\
Haber realizado una autoevaluación para el enriquecimiento del trabajo. & 4,7 & 5 \\
\hline
\end{tabular}

Utilización de escalas graduadas para autoevaluar y coevaluar: una experiencia de aula en la educación 
En las preguntas abiertas se reveló que el conocimiento de los criterios de valoración del curso y del TFEpP a través de la EG posibilitó una transparencia en la forma de evaluar que permitió reducir la ansiedad y fortalecer el proceso de coevaluación $\mathrm{y}$ autoevaluación para enriquecer tanto el TFEpP como la adquisición del aprendizaje. Al respecto de la coevaluación como actividad facilitadora del TFEpP se valoró como enriquecedora tanto en su etapa de realización como en la de recibimiento: "Realizar y recibir la evaluación de un compañero usando la escala me permitió ver la producción y el punto de vista de otro, que se encuentra haciendo el mismo aprendizaje que yo, en los mismos aspectos".

Al respecto de la autoevaluación se explicitó como relevante en el proceso de autorreflexión del trabajo realizado: "La actividad de autoevaluación con el uso de la escala me sirvió para cuestionarme mi trabajo desde otros puntos de vista".

Esta experiencia deja las puertas abiertas a seguir mejorando en las propuestas de actividades de evaluación formativa que fomenten la apropiación y protagonismo de los estudiantes en la educación virtual tanto en su proceso de aprendizaje, como en la certificación de éste.

\section{Referencias}

López-Pastor, V.; Pérez-Pueyo, Á.; Barba, J. \& Lorente-Catalán, E. (2016). Percepción del alumnado sobre la utilización de una escala graduada para la autoevaluación y coevaluación de trabajos escritos en la formación inicial del profesorado de educación física (FIPEF). Cultura, Ciencia y Deporte, 11 (31), 37-50.

Marsicano, I. (2018). Evaluación formativa, aprendizaje colaborativo y habilidades transversales: una experiencia de aula en la educación virtual. Reloj de agua, 18, 5-14.

Pérez-Pueyo, Á. \& Sobejano, M. (2017). Elaboración de instrumentos (escalas de valoración y graduadas) para la evaluación formativa. Revista Infancia, Educación y Aprendizaje, 3(2), 808-814. 